\title{
MÉTODO DE INTERPRETACIÓN Y ANÁLISIS HOLÍSTICO EN CASOS BIOÉTICOS
}

\author{
Ludwig Schmidt*
}

\begin{abstract}
Resumen: Este artículo propone un “método" fenomenológico con comprensión hermenéutica, que parte de un paradigma antropológico y ético y permite sistematizar e interpretar diversas situaciones en tres estadios: planteamiento, valoración (social, técnica, jurídica y bioética) y conclusiones e implicaciones del dilema. El esquema constituye un camino racional que facilita a todos los actores llegar al objeto propio de su disciplina desde una pluralidad de posturas y en forma integral y objetiva. Estudia y deduce por medio de un proceso lógico de concatenación sistémica de ideas, formas y experiencias, respetando las creencias y culturas presentes y estableciendo los mínimos éticos.
\end{abstract}

Palabras clave: método, juicio, deliberación, estudio de casos, educación, comité, bioética

\section{INTERPRETATION METHODS AND HOLISTIC ANALYSIS OF BIOETHICS' CASES}

\begin{abstract}
The author of this report offers a phenomenological method together with an hermeneutic comprehension which stems from an anthropological and ethic paradigm that allows to systematize and interpret different situations in three stadiums: planning, appraisal (social, technique, juridical and bioethics') and the dilemma's conclusions and implications. The scheme constitutes a rational way that helps all actors to arrive at the proper object of their discipline starting from a plurality of positions and in an integral and objective manner. It studies and derives, by means of a logical process of a systemic concatenation of ideas, forms and experiences, respecting the beliefs and cultures that are present and establishing the minimum ethics.
\end{abstract}

Key words: method, judgement, deliberation, case studies, education, committee, bioethics'

\section{MÉTODOS DE INTERPRETAÇÃO E ANÁLISE HOLÍSTICA NOS CASOS BIOÉTICOS}

Resumo: Este artigo propóe um "método" fenomenológico de compreensão hermenêutica, que parte de um paradigma antropológico e ético e permite sistematizar e interpretar diversas situaçôes em três fazes: fundamentação, valorização ( social, técnica, jurídica e bioética) e conclusóes e implicaçóes do dilema. O esquema constitui um caminho racional que facilita a todos os atores chegar ao objeto próprio de sua disciplina desde uma pluralidade de posturas de forma integral e objetiva. Estuda e deduz por meio de um processo lógico de concatenação sistêmica de idéias, formas e experiências, respeitando as crenças e culturas presentes e estabelecendo os mínimos éticos.

Palavras chave: método, julgamento, deliberação, estudo de casos, educação, comitê, bioética.

\footnotetext{
Vicepresidente del Centro Nacional de Bioética de Venezuela. Profesor de la Universidad Católica Andrés Bello, Caracas, Venezuela Correspondencia: 1schmidt@ucab.edu.ve
} 
"Es necesario el método para seguir tras la verdad de las cosas.

Esta regla no expresa el lugar común de que una ciencia debe tener también su método, sino que quiere decir que el procedimiento, esto es, el modo como estamos en general tras las cosas (méthodos), decide de antemano sobre lo que encontramos de verdadero en las cosas.

El método no es una pieza de la indumentaria de la ciencia entre otras, sino la instancia fundamental a partir de la cual se determina lo que puede llegar a ser objeto y cómo puede llegar a serlo"(1).

\section{Introducción $^{1}$}

Uno de los procesos más complejos que enfrentan algunas personas en su quehacer diario o en momentos críticos es decidir sobre la vida (propia y sobre todo de terceros), la salud de un ser, la calidad de vida y la protección del entorno ecológico. Determinar si se desconecta el respirador a un paciente o no, si es mejor abortar un nasciturus, cuál es la mejor alternativa terapéutica para un paciente o cómo regenerar el ambiente contaminado por la acción del hombre son decisiones que se complican si se deben adoptar en grupo y lograrse en forma colegiada.

El propósito de este proyecto de investigación es alcanzar la mejora de la calidad del juicio en los comités de bioética $(\mathrm{CB})$, para obtener decisiones colectivas concertadas y colegiadas. La calidad del juicio reside en la adecuada y racional reflexión moral, basada en la interpretación de los dilemas y situaciones bioéticas que, por lo general, son complejas, emocionales y demagógicas.

¿Por qué es importante mejorar la calidad del juicio bioético? En bioética se han propuesto, desde 1978, una diversidad de métodos con énfasis en materia clínica, elaborados por médicos y para justificar su acto ante un paciente (sujeto vulnerable). Sin embargo, los CB son grupos de personas con una formación multidisciplinaria y con pensamientos plurales. La justificación de esta investigación es tanto teórica como metodológica y práctica; teórica, porque se emplean enfoques de filosofía analítica que permiten una observación y diálogo 2

1 El presente trabajo es una versión revisada y modificada de la conferencia dictada en el V Congreso Mundial de Bioética, Gijón 2007.

2 El pensamiento dialógico en la actualidad fue impulsado por M. Buber F. Ebner, F. Rozenzweig, J. Heinrichs, J. Splett, G. Marcel, K. Jaspers, R. Guardini, F. Gogarten, J. Haberlas y A. Cortina. lo más objetivos posible; metodológica, porque ofrece un método filosófico y holístico para las alternativas deliberativas, y práctica porque cada persona, desde su capacidad de percepción e interpretación, puede ofrecer, mediante la sistematización de sus ideas, una opinión individual.

Se propone un "método" fenomenológico y una comprensión hermenéutica que parte de un paradigma antropoético, deliberativo y crítico, el cual permite sistematizar e interpretar las diversas situaciones desde una ética de mínimos a una de máximos.

Esta propuesta se implementa a través de un método científico que consta de tres estadios: (a) ver-planteamiento, (b) juzgar-valoración desde diversos puntos de vista (social, técnica, jurídica y bioética) e interpretación holística, y (c) actuar-las respectivas conclusiones e implicaciones del dilema.

El método bioético viene configurándose desde 1978 -con el Informe Belmont- hasta nuestros días. De esta manera, se han diseñado diversos procedimientos a partir del principialista (Beauchamps y Childress); otros partieron del casuístico (Jonsen, Siegler y Winshade), más tarde desde el narrativo (Beauchamps), pero los más difundidos fueron los clínicos o sincréticos, se mencionan a especialistas como D. Thomasma (1978), E. Pellegrino (1978), C. Candee y B. Puka (1983), L. McCullough (1984), G. Graver y D. Thomasma (1985), C. Strong (1985), H. M. Sass (Protocolo de Bochum, 1987), investigadores del Hasting Center (1988), C. Viafora (1989), J. Drane (1989) y D. Gracia (1991).

El que se propone es un método casuístico, sincrético y holístico. Obviamente, uno más pero no excluyente. Amplía la percepción y comprensión del dilema y viene desarrollándose desde 1994 para que diversas personas, con múltiples profesiones, puedan tomar decisiones éticas. Fue adoptado en los cursos de posgrado en bioética del Centro Nacional de Bioética de la Facultad de Medicina en la Universidad Central de Venezuela (CENABI-UCV) y en los cursos de bioética y derecho de la Facultad de Derecho de la Universidad Católica Andrés Bello (UCAB).

Para la secuencia se propone un proceso inductivo, el cual parte de: (a) la observación objetiva e integral de los hechos (ver), en la cual se analiza a cada actor involucrado en la situación, sus relaciones y su comportamiento en los escenarios en que participa; (b) la valoración 
ética del dilema o trilema que se da lugar en caso y que requiere de una colimación de criterios (juzgar) según los valores, principios y normas (objetivas y subjetivas) disponibles, para luego (c) emitir un juicio que oriente al consultante (actuar) y proponer una mejora ética como colofón de la opinión o dictamen ofrecido.

¿Con qué medios? A través de los datos objetivos suministrados y observados (evidencias), la información disponible para su consulta, la experiencia de estudios anteriores, el conocimiento multidisciplinar de los miembros del comité, las competencias personales (análisis y síntesis de situaciones, mentalidad abierta para percibir y comprender la realidad y otros puntos de vista, role playing, etc.) y el diálogo tolerante.

El proceso de toma de decisión no es trivial. Parte de la concienciación ética que los miembros del CB requieren en su ejercicio profesional ${ }^{3}$ y se desarrolla a través de un proceso de práctica (acción-reflexión) ${ }^{4}$. En otras palabras, la persona es capaz de visualizar los valores que entran en juego en cada caso (lo concreto) y, gradualmente, va apropiándose de criterios para enjuiciar sobre la base de normas (lo deontológico) y de principios-guía (lo teleológico), o para determinar las consecuencias y secuelas de sus acciones. El desarrollo ético es un proceso evolutivo y progresivo de adaptación al conflicto, a la problematización de situaciones. Como todo aprendizaje, los valores se desarrollan a partir de las prácticas sociales en que los seres humanos se relacionan y participan.

\section{Metodología}

El abordaje de estos temas requiere del desarrollo de ciertas capacidades de deliberación, valoración y toma de decisión ética. Se propone que sea en tres fases: (a) los profesionales o miembros del CB serán capaces de identificar sus valores y principios en sus propios criterios de "lo ético"; (b) serán capaces de comparar, analizar y evaluar los anteriores, contrastándolos con los valores y principios morales objetivos, y (c) internalizarán, concienciarán e integrarán los principios morales objetivos en la toma de decisiones.

3 El desarrollo ético de una persona se identifica con ella misma y con la institución a la cual presta servicios profesionales. En otras palabras, su pertenencia a una comunidad cuyos fines, intereses, valores y normas apropia como suyos.

4 La formación ética está influenciada por otros seres humanos particulares, los prójimos que son especialmente significativos al individuo.
De la justificación surge la necesidad de que en un $\mathrm{CB}$ se analice e interprete un caso dilemático en forma sistemática, es decir, que se disponga de una guía y forma homogénea de presentación, sobre todo para su revisión y análisis histórico, ético y jurídico. Para ello se persigue lograr:

- Aspectos que mejoren la toma de decisiones éticas en los planos personal y colectivo, concertado y colegiado.

- Una guía estructurada, sistemática y racional que permita a todo miembro de un CB deliberar y decidir consensual y colegiadamente.

- Competencias para el estudio y el proceso lógico de concatenación sistémica de ideas, formas y experiencias, respetando las creencias y culturas y estableciendo como punto de partida los mínimos éticos.

- Capacidad para dialogar desde una pluralidad de posturas, en forma interdisciplinaria, integral y objetiva.

- Decisiones éticas que se podrán enriquecer progresivamente sobre la base de la historia y las creencias del sujeto-objeto de estudio a los máximos éticos propios de su mundo existencial.

Lo anterior se alcanza mediante los siguientes objetivos operativos:

- Confrontar el dilema moral tratado.

- Detectar los hechos relevantes, actores, relaciones y naturaleza del dilema.

- Aclarar y definir la terminología empleada.

- Identificar los valores en los propios sentimientos de justicia, que se relacionan con las actitudes $\mathrm{y}$ acciones detectadas y que se establecen en las diversas posturas individuales.

- Formular los principios morales en palabras propias del individuo, a partir de los valores anteriores.

- Evaluar los propios valores y principios a la luz de los valores y principios objetivos.

- Determinar los criterios de juicio que fundamentan las posturas sobre dicho dilema ético-profesional.

- Integrar principios morales objetivos en la toma de decisiones.

- Evaluar la evolución histórica del planteamiento moral.

Igualmente, se consideran objetivos académicos específicos, adicionales para la concienciación individual, los siguientes: 
- Ampliar y ejercitar su capacidad de razonar (extraer conclusiones de distinto tipo, clasificar y categorizar, trabajar con la coherencia y la contradicción, formular preguntas, identificar supuestos, formular relaciones causa-efecto, conocer y evitar -o saber utilizar- la vaguedad en el lenguaje, distinguir ambigüedades, reconocer la interdependencia entre medios y fines, definir términos, identificar y utilizar criterios, ejemplificar, construir hipótesis, contextualizar, anticipar, predecir y estimar las consecuencias, generalizar, descubrir falacias no formales, normalizar frases del lenguaje cotidiano).

- Desarrollar el pensamiento creativo.

- Familiarizar a cada miembro del CB con la práctica de la deliberación, la tolerancia y la prudencia.

- Expandir su capacidad individual para encontrar sentido en la experiencia (descubrir conexiones y alternativas, relaciones parte-todo y todo-parte, detectar y reconocer incoherencias, ofrecer razones).

El método se contempla desde una postura multirreferencial y multivariada, con respeto a la intencionalidad de los actores, los fines y medios en juego y las posibles consecuencias de dicho acto.

\section{Resultados}

El uso del método ha demostrado una mejora sustancial en la capacidad de percibir, analizar, interpretar y decidir situaciones éticas. Las experiencias en más de 50 seminarios y talleres de análisis e interpretación de casos bioéticos en estos últimos 12 años, en los que se prepararon más de un millar de miembros de $\mathrm{CB}$ y futuros profesionales de Derecho, Medicina, Odontología, Filosofía y Teología, han mostrado resultados que, cualitativa y cuantitativamente, demuestran una sustancial mejora en la rapidez y calidad de las decisiones éticas, después de los primeros ejercicios con el método propuesto. Obviamente, no todos han participado de la investigación formal: sólo desde 1996 se iniciaron las investigaciones docentes en forma gradual hasta 2006.

La investigación se fundamenta en la Teoría del Desarrollo Humano de James Fowler, las indicaciones metodológicas de Eduardo Schmidt(2) y la experiencia docente señalada anteriormente. Los criterios para un adecuado juicio contemplaron: (a) la forma del razonamiento lógico empleado por el individuo (basada en Piaget); (b) su perspectiva ecosocial (basada en Selman); (c) la forma como cada persona hace sus juicios morales (basada en Kolhberg); (d) el lugar donde se ubicó la autoridad para darle sentido a su vida; (e) la forma como da coherencia al mundo en que vive, $y$ (f) el modo como maneja la función de lo simbólico. Todos estos factores se evaluaron antes y después del curso, mediante cuestionarios, y sus resultados se contrastaron. Luego, en el mismo curso, se evaluaron conocimientos y destrezas de pensamiento. Obviamente, esto se realiza con grupos de control que emplean enfoques y criterios diversos.

La exposición sistemática propuesta para el estudio de dilemas bioéticos está centrada en el proceso de la decisión moral, en el cual la persona interioriza y se apropia del conocimiento moral mediante una serie de operaciones intencionales y conscientes. Dicho proceso está enmarcado en el concepto de la "autoconciencia" del decisor (cada miembro del CB), para lo cual se propone la estructura descrita en la figura 1. 
Figura 1: Método propuesto en su forma integral.

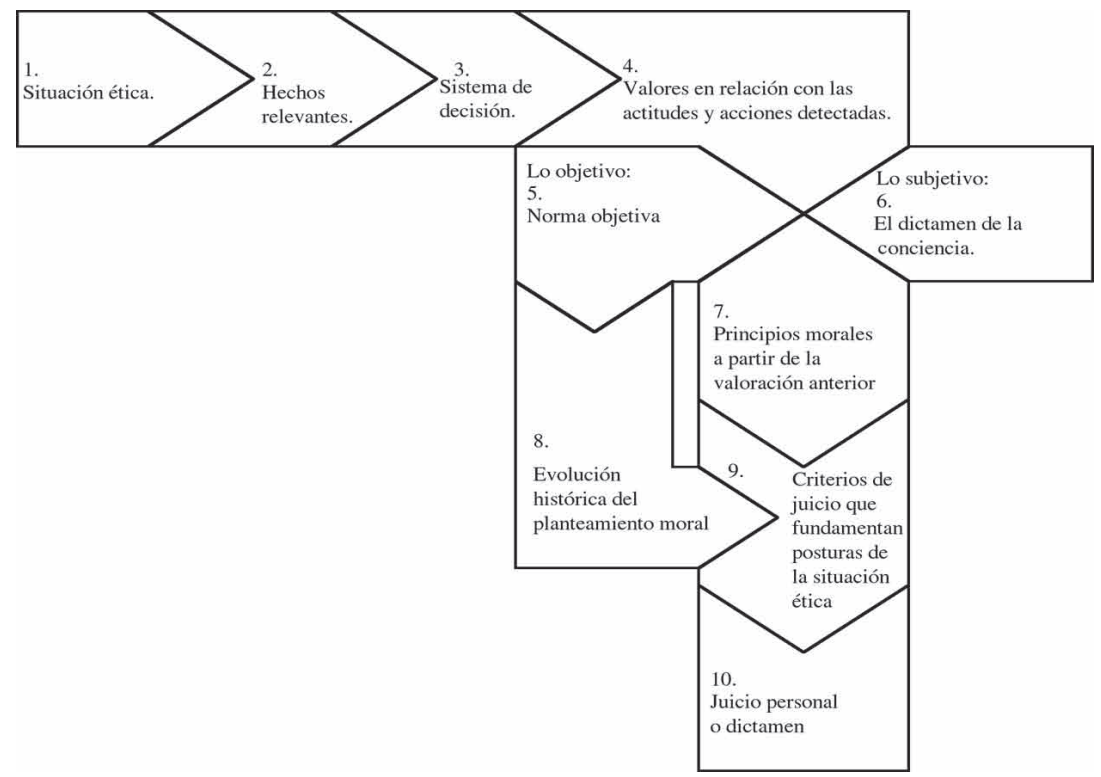

En el primer estadio del proceso, el sujeto comienza a apropiarse de los datos del dilema moral. El conocimiento de éste le permite "experimentar" en este primer nivel de operación consciente e intencional al identificar el objeto (el sistema ético y los valores presentes). Este estadio corresponde al discernimiento de la realidad, en especial del dilema bioético que está considerando y permite:

1. Confrontar el dilema bioético tratado: en lo posible desde "diversas ópticas o puntos de vista" y lo más amplia o extensamente posible. De esta "lectura" se presta una aguda atención a los datos del objeto por conocer. Este paso preliminar permite despertar la sensibilidad ética de los sujetos a las situaciones particulares del objeto de estudio.

2. Detectar e identificar los hechos relevantes del dilema bioético y su secuencia. En otras palabras, aquellos que clarifican, agrupan o identifican mejor el dilema. Destacar el hecho relevante fija la atención en el problema central del dilema moral tratado. Es tan importante la adecuada recopilación de datos como su adecuada correlación para captar su inteligibilidad.

3. Análisis del sistema de referencia o de decisión ética: actores, relaciones, escenarios y naturaleza del dilema moral (premisas ontológicas y éticas). Ello permitirá reconocer el entorno, las interacciones y las comunicaciones existentes. Además, se recomienda definir la terminología empleada (si aplica).
4. Identificar los valores en sus propios sentimientos de justicia: para que el sujeto pueda emitir juicios morales es necesario desarrollar en él la capacidad de reflexión, de expresión de sus propios sentimientos y de cómo éstos influyen en sus juicios éticos. Sólo así tendrá la apertura necesaria para tomar una postura de sana crítica frente a su comportamiento cuando se deja llevar por sus sentimientos de justicia. Por "valores morales" nos referimos a "conceptos que indican algún objeto apreciado como bueno, valioso o deseable" $(2,3)$, que se relacionan con las actitudes y acciones detectadas y que se establecen en las diversas posturas individuales. Los datos de "valor objetivo" permiten hablar de un nivel empírico en el mundo interior del sujeto analizado, para estudiarlo racional y sistemáticamente desde su contexto o "escenario(s)" y las "relaciones" que dentro del mismo presenta el fenómeno ético en cuestión.

En un segundo estadio, la experiencia del objeto y la recopilación de datos no es todo el conocimiento, sino apenas parte del mismo. Es necesario "entender" el dilema moral tratado, para lo cual se deberá intensificar en el sujeto su conciencia intencional. Entender es unificar y correlacionar los datos del objeto o captar sus relaciones y funciones. Además, se debe reconocer la moralidad del dilema en cuestión. La moralidad surge de una dimensión objetiva (la norma) y otra subjetiva (la conciencia). Ambas son mutuamente recurrentes, 
ya que si la primera no transfiere sus contenidos a la segunda la generación de conocimiento se estanca, y si la segunda pretende prescindir de la primera carecerá de la función antropológica, social, técnica, jurídica y teológica.

5. Lo objetivo. La norma objetiva o ley moral comprende desde la ley divina (ley natural y ley divina positiva) hasta la ley humana. Según sea el caso, se ilumina y valora la situación desde la Sagrada Escritura, el Magisterio de la Iglesia (en el caso de estudios de ética teológica) o cualquier otra creencia que practique el sujeto-objeto de estudio. Aunque primero se busca determinar el ordenamiento jurídico, deontológico y social vigente de la sociedad (en ética civil, la norma reduce su campo de entendimiento a la ley positiva humana). A lo largo de la historia del hombre se ha establecido en cada cultura un marco de doctrina, de legislación y de jurisprudencia. Dicho marco permite ubicar mejor el dilema moral objeto de estudio.

6. Lo subjetivo. La norma subjetiva posibilita también reconocer la moralidad del (de los) acto(s) del dilema tratado. Generalmente, se vincula con sus actitudes y creencias. Establecer lo que dicta la conciencia, en particular, la conciencia intencional del actor de la situación, sólo es posible en forma parcial y a través de sus actos: sus "obras" ilustran el sentido y la dirección de su opción fundamental.

Después de "experimentar y entender", es preciso juzgar acerca de lo experimentado y aprendido, discriminar si lo entendido corresponde realmente a lo experimentado. Obviamente, de los "hechos" y de la "norma" se pasa a un nivel más de intensificación. El juicio ha de decidir dialécticamente por el "si" o por el "no". La operación de juzgar es un acicate para evitar el divorcio entre el conocimiento y la realidad. El paso previo al juicio de la conciencia moral requiere de:

7. Formular los principios morales en palabras propias, a partir de los valores anteriores. Evaluar los propios valores a la luz de los valores objetivos. Para ello se deberá tener presente que sólo la conciencia recta y cierta es norma incondicional de la opción moral. En caso de duda, se debe buscar la certeza con la ayuda de los sistemas éticos.

8. Evaluar la evolución histórica del planteamiento moral. La experiencia histórica y cultural es de gran importancia para comprender el significado y los valores presentes en el hecho analizado (todo ello en caso de corresponder a un problema cuya experiencia histórica enriquezca el significado moral del objeto de estudio).

9. Determinar los criterios de juicio basados en los puntos anteriores. Esto exige sopesar las evidencias en favor o en contra de lo entendido o, dicho de otra manera, verificar sus condiciones de verdad.

Todo lo anterior permite evaluar con criterios objetivos las propias intuiciones y principios morales $(2, p$. 197 $198)^{5}$, dejando la postura individual para la próxima etapa. Aunque no conduce a una acción inmediata, se puede orientar el acto moral o el consejo a quien lo solicite. Con el punto 9 se concluye la sistematización para el estudio ético en forma objetiva; sin embargo, siempre existen dudas o inquietudes, posturas o críticas individuales adicionales a las expuestas.

10. Dictamen personal. Fundamenta las posturas sobre el dilema moral e integra los principios morales objetivos en la toma de decisiones. Fruto de todo lo anterior se puede cerrar el proceso con una decisión documentada, reflexionada y sistematizada adecuadamente.

Para concluir, se recomienda la elaboración de una propuesta de mejora, una enseñanza o moraleja que encierra la respuesta del cómo debió haber sido o lo que se recomienda que sea.

El siguiente proceso de sistematización permite concienciar la situación en contextos cada vez más amplios o abstractos, al romper con una visión restringida del dilema moral y reflexionar sobre la significación de la vida, las creencias, el bienestar y la calidad de vida de la persona y de su entorno, al documentarse sobre una experiencia histórica, antropológica y ética. A través de la concienciación se logra:

- Despertar, desarrollar y sensibilizar una conciencia crítica sobre el valor de la vida humana.

- Reconocer el respeto por la dignidad y la libertad del sujeto.

- Establecer la necesidad de una praxis más activa y vinculante con el entorno ecosocial, político, económico y tecnológico.

- Evaluar el quehacer moral.

5 Es importante la capacidad de autocrítica frente a lo que siempre se ha aceptado. Permite reforzar y esclarecer muchas intuiciones y principios moralmente correctos, sobre todo si éstos han sido asimilados en forma distorsionada o errónea. 
La moral del individuo coincide con la actuación y alcance de su propia conciencia. Por tanto, se trata de una conciencia testigo y juez de la adecuación del acto a la ley, la cual se ensancha notablemente hasta el punto de convertirse en núcleo de control, diagnóstico y "convicción moral", en el sentido del pensamiento paulino. La persona tiene que desarrollar su identidad moral, comprender su responsabilidad y compromiso con el entorno, con los demás y consigo misma.

Es importante destacar la existencia del argumento de la "pendiente resbaladiza" o "efecto dominó", un clásico argumento consecuencialista que se aplica donde se pone en juego la vida de una de las partes. Una vez legalizada una medida, en casos de solicitud voluntaria, el clima social conduce a médicos y familiares a deslizarse hacia su aplicación en casos de enfermos inconscientes o incapaces que no han expresado su autorización. Ha sido criticado por poca rigurosidad en los razonamientos previos. Sin descartar su uso, se recomienda una adecuada claridad en los enunciados y la lógica empleada.

\section{Discusión}

La sistematización puede validar el conocimiento mediante un ciclo teórico y otro aplicado. El primero (paradigma) permite determinar si existe consistencia intelectual entre los principios, criterios y contrapartes verificativas y aquellas susceptibles de explicación o dimensión prospectiva del fenómeno; el segundo tiene eficacia pragmática que implanta los resultados verificativos. Allí se demuestra la coherencia del conocimiento objetivo y si la información sistematizada puede ser obtenida. De esta manera, y desde una comprensión ética de mínimos, se dispondrá de una base sólida para emitir un juicio. La opinión personal será contrastada con los demás miembros del CB y se formulará posteriormente el juicio concertado y colegiado.

$\mathrm{Al}$ igual que se aprende a hablar a partir desde el abecedario hasta la compleja labor de hacer frases coherentes y claras, el proceso de investigación bioética requiere ir avanzando desde patrones simples para que evolucione epistemológicamente. Por ejemplo, es necesario desarrollar(4):

- La sensibilidad temporal. Organizar las percepciones en relación con el pasado, presente y futuro, a corto, mediano y largo plazo. Las consecuencias y secuelas de las acciones; las presiones que el pasado ejerce sobre el presente y el futuro; la visualización de los efectos probables del presente sobre el futuro y viceversa; los antecedentes de los acontecimientos presentes en la historia y el índice de cambio con el tiempo.

- La conceptualización. La conciencia del problema es, probablemente, el resultado más importante de la capacitación metodológica. Consiste en identificar las partes y actores, sus relaciones y escenarios, los objetivos que los vinculan y el propósito que persiguen.

- La distribución de patrones cognoscitivos. La organización es la aplicación de un patrón a un conjunto de situaciones dadas; pensar es crear y utilizar patrones. La existencia de patrones disponibles para el entorno es un capital intelectual. Se propone el empleo de herramientas de expansión o búsqueda (brainstorming), de visualización, de categorización, de relación, de contracción o síntesis, de causa-efecto, de juego de roles, de jerarquización, entre otras.

- El análisis. Dividir los conjuntos en pares y los procesos en etapas constituye un proceso fundamental del pensamiento y es el que tradicionalmente se ha aprendido: el fraccionamiento racional y lógico en estructuras simples de arreglos complejos y la identificación de denominadores comunes en diversas situaciones. Se mencionan cuatro tipos de procesos analíticos importantes: descubrir una regla para hacer idóneo un ejemplo; ilustrar una regla por medio de un ejemplo tomado de la experiencia; producir normas alternativas para que se ajusten al mismo conjunto de acontecimientos y abstraer o modelar secuencias complejas de interacciones, incluyendo la realimentación.

- El empleo de la experiencia. La comparación admite ilustrar los patrones mediante observaciones directas o indirectas, ya sea por contención, asociación, solapamiento o por categorías antagónicas.

- El uso de aseveraciones condicionantes y contrarias a los hechos. Aprender a utilizar esas proposiciones y comprobarlas adecuadamente es parte importante en la preparación intelectual de los críticos y se requiere de un adiestramiento sistemático.

- La orientación hacia la intervención. La realidad condiciona el pensamiento crítico y la capacidad de manejar las situaciones a través del empleo de argumentaciones sólidas y bien fundadas es esencial para entrar en acción en el mundo real. 
Método de interpretación y análisis holístico en casos bioéticos - Ludwig Schmidt

- La conciencia de los costos y de los beneficios de una acción. No sólo costos económicos sino también emocionales, sociales y tecnológicos. Este proceso, aunque contingente a la conciencia, es importante para la adecuada ponderación de las acciones y las alternativas posibles.
El método esbozado permite, mediante el empleo de herramientas cognoscitivas, el pensamiento práctico y la sistematización de las ideas en la deliberación de casos bioéticos o situaciones éticas complejas. Su empleo en sesiones de $\mathrm{CB}$ amplía las percepciones individuales, mejora el razonamiento y genera decisiones consensuadas y colegiadas.

\section{Referencias}

1. Heidegger M. La pregunta por la cosa. Buenos Aires: Alfa Argentina; 1975: 93.

2. Schmidt E. Moralización a Fondo. Lima: Universidad del Pacífico; 1993: 57-119.

3. Cavanagh GF, McGovern AF. Ethical Dilemmas in the Modern Corporation. Englewoof Cliffs NJ: Prentice Halls; 1988: 14.

4. Meehan EJ. Introducción al pensamiento crítico. México: Trillas; 1975: 20-25.

Recibido: 25 de diciembre de 2007

Aceptado: 22 de enero de 2008 